\title{
Probiotic Bacteria for Healthier Aging: Immunomodulation and Metabolism of Phytoestrogens
}

\author{
José María Landete, Pilar Gaya, Eva Rodríguez, Susana Langa, Ángela Peirotén, \\ Margarita Medina, and Juan L. Arqués
}

Departamento Tecnología de Alimentos, Instituto Nacional de Investigación y Tecnología Agraria y Alimentaria (INIA),
Carretera de La Coruña Km 7, 28040 Madrid, Spain

Correspondence should be addressed to Juan L. Arqués; arques@inia.es

Received 2 February 2017; Revised 11 April 2017; Accepted 23 August 2017; Published 1 October 2017

Academic Editor: Gjumrakch Aliev

Copyright (C) 2017 José María Landete et al. This is an open access article distributed under the Creative Commons Attribution License, which permits unrestricted use, distribution, and reproduction in any medium, provided the original work is properly cited.

\begin{abstract}
Age-related degeneration gives rise to a number of pathologies, many of them associated with imbalances of the microbiota and the gut-associated immune system. Thus, the intestine is considered a key target organ to improve the quality of life in senescence. Gut microbiota can have a powerful impact in the deterioration linked to aging by its nutritional and immunomodulatory activity. Reduced numbers of beneficial species and low microbial biodiversity in the elderly have been linked with pathogenesis of many diseases. A healthy lifestyle with an elderly customized diet including probiotics can contribute to reducing the chronic proinflammatory status and other age-related pathologies. Beneficial effects of probiotic lactic acid bacteria and bifidobacteria to alleviate some of these disorders based on their immunomodulatory properties as well as their capacity to produce bioactive metabolites from dietary phytoestrogens are summarized. On one hand, the preservation of gut barrier integrity and an increased ability to fight infections are the main reported immune benefits of probiotics. On the other hand, the intake of a diet rich in phytoestrogens along with the presence of selected probiotic bacteria may lead to the production of equol, enterolignans, and urolithins, which are considered protective against chronic diseases related to aging.
\end{abstract}

\section{The Aging Process}

The time-dependent biological complex processes that produce a gradual generalized deterioration of the anatomy and physiological functions of organisms are defined as aging. It led to weakness to environmental stress and therefore increases the risk of disease and death. Among multicellular organisms, aging is marked by a progressive decline in the function of multiple cells and tissues. Apparently, the event of aging is genetically determined and modulated by the environment, but the causes of those irreversible changes are still an unresolved challenge. Understanding aging is an important objective that may help to modify the aging process or the senescence effects. The aging rate could be determined by two major circumstances: the accumulation of damage and the effectiveness of somatic maintenance mechanisms [1]. Nine cellular and molecular hallmarks of aging have been proposed by López-Otín et al. [2], which are genomic instability, telomere attrition, epigenetic alterations, loss of proteostasis, deregulated nutrient sensing, mitochondrial dysfunction, cellular senescence, stem cell exhaustion, and altered intercellular communication. In human cells, the presence of telomerase suggests that cells may be programmed to undergo senescence as a mechanism to "count" cell divisions, although stress and damage accumulation are also important for the telomere shortening [3].

The main aim of aging research is to improve the quality of life. Age-related degeneration gives rise to a number of pathologies, such as osteoarthritis, atherosclerosis, lung emphysema, malignancies (gastrointestinal, prostate), and dementias. The aging process is dependent on antistress responses, which act as antiaging mechanisms. Furthermore, immunosenescence, which can be defined as a decline in the functionality of the immune system, contribute to a chronic state of basal inflammatory activity (inflammaging) [4-6]. 
The most studied and reproducible nongenetic intervention in aging research is dietary restriction. However, the importance of diet composition has been highlighted when applying a reduction in calorie intake to regulate the lifespan [7]. Another important factor that can play a key role in senescence is the impact of the diet on the gut microbiota composition and the chronic inflammation. Thus, age-related changes in the nutritional behaviour are associated with the imbalances of the microbiota and the gut-associated immune system. A healthy lifestyle with an elderly customized diet including probiotics can contribute to reduce the chronic proinflammatory status and other age-related pathologies [8$10]$.

1.1. Aging and Gut. The gastrointestinal (GI) tract is characterized by its complexity, being the main and largest site for interaction with the external environment. The GI tract is covered by a single layer of epithelial cells, which are responsible for the digestion and absorption of nutrients and electrolytes, as well as homeostasis. Moreover, the gut-associated lymphoid tissue provides an important first line of defence that controls the equilibrium between tolerance and immunity against orally acquired food and microbes. The human gut contains the enteric microbiota, whose mutualistic relationship contributes to the maintenance of health, including digestion of complex carbohydrates, intestinal homeostasis, synthesis of essential nutrients and vitamins, protection against pathogens, and stimulation of the immune system [11]. Age-associated modifications of the gut cause disorders that clearly affect the quality of life of elderly population, becoming a major cause of morbidity [12].

A distinguishing characteristic of the aging gut is the overexpression of proinflammatory cytokine IL-6, which has an effect on the intestinal barrier function and mucosal immune system [13]. Persistence of inflammaging can also facilitate cancer development and progression [6, 14]. During postmenopause/andropause periods IL-6 levels are increased. Overexpression of IL-6 might have important ramifications with regard to both impaired immunity and intestinal barrier integrity, which can downregulate innate immunity to pathogens and consequently increase the susceptibility to infections in the elderly. Moreover, those changes in the intestinal permeability could be crucial in the development of local (celiac disease, colorectal cancer, or inflammatory bowel disease) and systemic diseases (diabetes, chronic heart failure, or obesity) and even in central nervous system disorders $[15,16]$.

Physical and immunological impairments of intestinal barrier are correlated with age-related diseases and lifespan. The cross-talk between gut microbiota and the gut-associated lymphoid tissue has a powerful effect on the host immune response which can lead to systemic metabolic effects [17]. Thus, the intestine is a key target organ to improve the quality of life in senescence $[18,19]$.

1.2. Impact of Gut Microbiota on Aging. Alterations in morphology and physiological functions modify the physical environment of the elderly gut, which affect the composition of the intestinal microbiota. Moreover, antibiotics are still an irreplaceable therapy for the elderly, which have also a huge influence on the intestinal microbiota composition. Dysbiosis is associated with various metabolic, infectious, and inflammatory disorders including malnutrition, diabetes, bowel diseases, Clostridium difficile infections, obesity, colon cancer, and atherosclerosis [20,21]. An interesting clue to unravel the role of gut microbiota in some aged-related diseases is the big interindividual variations among older subjects compared to the adults $[8,22]$.

Gut microbiota has a strong impact in human physiology and, therefore, on the health status in the elderly and age-related diseases [23]. Its immunomodulatory properties could help in two main aspects of aging as immunosenescence and inflammaging. Aging can be considered as an immune disorder [24]. Commensal bacteria can modulate the host inflammatory response, mainly by targeting NF$\kappa \mathrm{B}$. It has been proposed that an increased presence of IL6 -inducing bacteria in the elderly could be associated with elevated intestinal levels of IL- 6 in the gut and therefore at systemic level [14]. Thus, an aged-type microbiota shows a low microbial biodiversity, enriched in pathobionts and facultative anaerobes and depleted of Firmicutes, which is linked with an increase of proinflammatory signals [22, 25-27]. Another important aspect to address during the aging process is the interaction between the microbiota and the metabolism of dietary components and their potential beneficial effects in the generation of bioactive nutrients $[28,29]$.

Host age, health status, and environmental factors can modulate our microbiota composition. Improving the profile of the gut microbiota during human aging, mainly lifestyle factors and nutritional habits, would have an impact on human health and longevity since longevity process is associated with human gut microbiota changes [30]. The role of gut microbiota in human aging include two main aspects: immunomodulatory and nutritional (energy availability and metabolism). Dietary interventions with probiotics or fecal bacteriotherapy could be employed to rationally enrich the gut microbiota of the elderly [20,30-33].

\section{Potential Beneficial Effects of Probiotics on the Aging}

Probiotics can be applied to modulate the age-related gut microbiota imbalance and to introduce strains with specific health-promoting effects. The principal claimed benefits of probiotics in elderly people are prevention of diarrheal diseases, protection against pathogens, enhancement of the intestinal barrier function, improvement of gastrointestinal motility and inflammatory intestinal disorders, immunomodulatory effects, and prevention of colon cancer $[34,35]$.

Probiotic intervention, with or without a specific diet composition, would help to improve the microbiota functionality in order to obtain health benefits during the old age. In this context, a diet rich in phytoestrogens can be considered an interesting therapeutic approach against aging due to their estrogenic and antioxidant actions. Here we summarize two promising beneficial effects of probiotics to alleviate 
some age-related pathologies based on their immunomodulatory properties as well as their capacity to produce bioactive metabolites from dietary compounds, such us phytoestrogens.

2.1. Probiotics to Improve Immune-Health. Senescence is associated with a decline in immune function and an increase in inflammation [10]. The effects of IL-6 on intestinal permeability could increase the penetration of microbes and/or toxins into the body $[10,36]$. Probiotic intervention can improve some of these age-associated modifications of the immunological features [37-39]. However, despite their promising benefits, little is known about the effect probiotics on intestinal barrier and immune function.

Probiotics can exert beneficial effects on the preservation of gut barrier integrity and function stimulating the activity and growth of beneficial bacteria and regulating the expression of tight junction proteins [40-47].

Aging process affects innate immunity, with reduced activity or number of natural killer (NK) cells, and adaptive immunity, with reduced antigen-specific $\operatorname{IgA}$ antibody and cellular immune responses [48]. Probiotic treatments can ameliorate some of these processes modulating cytokine production, improving distribution and function of NK cells, macrophages, granulocytes, and T cells in the circulation, and enhancing mucosal and systemic antibody responses [49-51].

Lactic acid bacteria (LAB) and bifidobacteria are commonly found in the gut of humans and other animals as well as in probiotic supplements and foods. Their immunomodulatory properties can be applied in age-related disorders. Studies carried out on mice demonstrated the potential of probiotics to palliate the effects of aging on the immune system. Administration of Lactococcus lactis H61 or L. rhamnosus MTCC 5897 improved the age-associated Th1/Th2 imbalance [52, 53]. Bifidobacterium adolescentis BBMN23 and Bifidobacterium longum BBMN68 isolated from healthy centenarians enhanced both innate and acquired immunity in mice [54]. Supplementation of aged mice with the probiotic Lactobacillus paracasei NCC2461 improved the specific adaptive immune response, with higher IgG2a levels after antigenic challenge [55]. The strain L. rhamnosus CRL1505 was able to increase the peritoneal macrophages phagocytic activity and the number of intestinal $\operatorname{IgA}^{+}$cells in the intestinal mucosa of aged mice [56]. Recently, the effect of Lactobacillus plantarum WCFS1, L. casei BL23, and Bifidobacterium breve DSM20213 on gut barrier and immunity in accelerated aging mice was investigated. That study found that age-related decline in mucus and systemic immunity can be modulated by probiotics but also highlights the risk of translating the beneficial effects of probiotics observed in young animals or humans to the elderly [57].

Several human studies also show a higher ability to fight infections following probiotic consumption. Bifidobacterium lactis HN019 enhanced phagocytic activity and number of NK cells in elderly subjects $[51,58,59]$. A probiotic cheese containing Lactobacillus rhamnosus HN001 and Lactobacillus acidophilus NSFM increased the cytotoxicity of NK cells in elderly volunteers [60]. Administration of yogurt containing the probiotic strain Lactobacillus casei DN-114001 to elderly people reduced the length of winter infections compared to the control group [61]. Likewise, an improvement in the nutritional and immunological status of enterally fed elderly subjects was observed by the administration of a fermented milk containing Lactobacillus johnsonii La1 [62].

2.2. Probiotics, Phytoestrogens, and Aging. Phytoestrogens are polyphenols present in plants or foods derived from plants foods such as soya, flaxseed, cereals, vegetables, fruit, chocolate, and tea [63-65]. Phytoestrogens such as coumestans, stilbenes, ellagitannins, lignans, and isoflavones are similar to endogenous estrogen and therefore they have both antiestrogenic and estrogenic effects [66]. Intake of these compounds may be protective against chronic diseases related to aging, such as cardiovascular and bone diseases, various cancers, menopausal symptoms, and cognitive function [67-73]. These health benefits from phytoestrogens consumption should be attributed to the bioactive metabolites produced by gut bacteria and to the modulation of the intestinal bacterial population $[74,75]$. Thus, the intake of a diet rich in isoflavones (soybeans and soy derived foods), lignans (flax seeds, cereals, etc.), and/or ellagitannins (pomegranates, cherries, etc.) along with the presence of selected probiotic bacteria may ensure the production of equol, enterolignans, and urolithins in the gut, respectively [76-78] (Table 1). This approach should be considered in the prevention and improvement of aging-related pathologies.

The transformation of isoflavones, lignans, and ellagitannins by bacteria is an essential step because

(1) equol, enterolignans, and urolithins are more bioavailable than their respective dietary phytoestrogens $[79,80]$ (Figure 1),

(2) equol, enterolignans, and urolithins have more estrogenic/antiestrogenic activities than their precursors. The biological action of these derived compounds is mediated primarily by estrogen receptors [81], modulating hormone levels and expression of estrogen receptors $[82,83]$. They may act as anticarcinogens through antiestrogenic actions competing with estradiol to bind estrogen receptors [84]. Equol, enterolignans, and urolithins have various estrogenic effects in postmenopausal women, such as decreased plasma levels of estrone and estradiol sulfate and changes in the metabolism of estrogen (from 16 $\alpha$-hydroxylation to 2-hydroxylation, a less carcinogenic pathway) [85, 86],

(3) equol and enterolignans are more antioxidants than their precursors $[80,87]$, acting against DNA damage and lipid peroxidation. The antioxidant activities of enterolignans have also been suggested to contribute to the reduction of hypercholesterolemia, hyperglycemia, and atherosclerosis [88],

(4) finally, equol, enterolignans, and urolithins have antiinflammatory effects and exert antiproliferative and apoptosis-inducing activities $[89,90]$.

Although specific bacteria responsible for the equol, enterolignans, and urolithin production are still being investigated, 
TABLE 1: Potential probiotic strains implicated in the metabolism of phytoestrogen.

\begin{tabular}{|c|c|c|}
\hline Bacteria & Transformation/production & Reference \\
\hline Lb. rhamnosus CRL981 & Daidzin to daidzein & {$[100]$} \\
\hline Lb. plantarum CECT $748 \mathrm{~T}$ & Daidzin to daidzein & {$[80]$} \\
\hline Lactobacillus sp. Niu-O16 & Daidzein to dihydrodaidzein & {$[101]$} \\
\hline Lb. rhamnosus INIA P540 & Daidzin to dihydrodaidzein & {$[91]$} \\
\hline Ent. faecalis INIA P333 & Daidzin to dihydrodaidzein & [91] \\
\hline $\begin{array}{l}\text { Lb. mucosae EPI2, Ent. faecium EPI1, } \\
\text { Finegoldia magna EPI3, and Veillonella sp. } \\
\text { EP }\end{array}$ & Daidzein into equol & {$[110]$} \\
\hline Lactococcus garvieae 20-92 & Daidzein into equol & {$[112]$} \\
\hline B. breve 15700 and B. longum BB536 & Daidzein into equol & [113] \\
\hline B. adolescentis INIA P784 & Enterodiol production from flax seed & {$[78]$} \\
\hline $\begin{array}{l}\text { Gordonibacter urolithinfaciens and } \\
\text { Gordonibacter pamelaeae DSM } 19378 \mathrm{~T}\end{array}$ & Urolithin C from ellagic acid & [137] \\
\hline
\end{tabular}

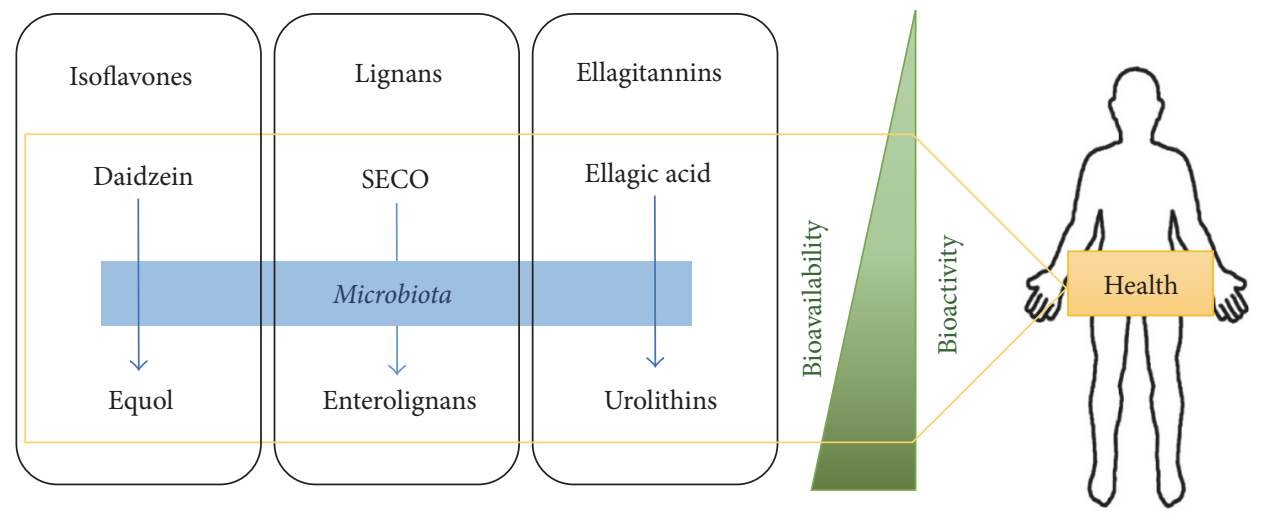

FIGURE 1: Isoflavones, lignans, and ellagitannins intake are metabolized by potential probiotic bacteria to produce equol, enterolignans, and urolithins, respectively. These compounds are more bioavailable and bioactive than their precursors.

some $\mathrm{LAB}$ and bifidobacteria have been involved in the metabolism of these compounds $[78,91]$.

2.2.1. Isoflavones, Aging, and Probiotic Bacteria. In soy and unfermented soy foods, isoflavones are as glycosides such as daidzin, genistin, or glycitin. These compounds are less estrogenic than their aglycones daidzein, genistein, and glycitein, respectively. Daidzin, genistin, or glycitin cannot be absorbed because of their higher molecular weights and hydrophilicity [92]. Then, their bioavailability requires the transformation in daidzein, genistein, and glycitein by means of $\beta$-glycosidase activities.

Benefits of soy in aging are derived from the isoflavones metabolism of bacteria, including protection against breast cancer [93], prostate cancer [94], menopausal symptoms [95], heart disease [96], osteoporosis [97], and cognitive function [98].

LAB and bifidobacteria are very important in the transformation of naturally occurring isoflavones in the form of $\mathrm{O}$-glucosides, $\mathrm{C}$-glucosides, or their methylated forms in the bioactive isoflavones daidzein and genistein and even in the formation of dihydrodaidzein [91]. The capabilities of converting daidzin to daidzein have been observed in Weissella confusa, Enterococcus durans $\mathrm{KH}$, and Lactobacillus paraplantarum KM [99], as well as in L. rhamnosus CRL981 [100]. Lactobacillus sp. Niu-O16, isolated from bovine rumen contents, converted daidzein to dihydrodaidzein [101].

Daidzein, genistein, dihydrodaidzein, and dihydrogenistein possess physiological properties of interest in healthy aging [68]. The production of daidzein and dihydrodaidzein facilitates the formation of equol and/or $O$-desmethylangolensin (O-DMA). Equol has enhanced effects due to its greater affinity for estrogen receptors, unique antiandrogenic properties, and superior antioxidant activity. In vivo and in vitro beneficial effects of equol have been demonstrated [102]. So, it has been possible to demonstrate in vitro the effect of equol against aging in skin [103] and nervous system [104]. On the other hand, the effect of equol in the improvement of menopause symptoms and in the prevention of cancers and cardiovascular diseases has been demonstrated both in vitro [105] and in vivo [106-108]. Evidence from in vitro studies suggests that $O$-DMA may have several cancer-related biological actions. However, results from human metabolic 
studies and observational studies of disease risk suggest that these actions may not be physiologically relevant in vivo due to the amount and form (primarily glucuronide) of circulating O-DMA [109].

A mix of bacteria composed of Finegoldia magna EPI3, Lactobacillus mucosae EPI2, Enterococcus faecium EPI1, and Veillonella sp. strain EP was able to transform daidzein into equol [110]. Similarly, anaerobic incubation of Eggerthella sp. Julong 732 and Lactobacillus sp. Niu-O16 transformed dihydrodaidzein to S-equol [111], although most of equolproducing microorganisms belonging to the Coriobacteriaceae family, Lactococcus garvieae 20-92 [112], B. breve 15700, and B. longum BB536, were also able to produce equol [113]. $\mathrm{LAB}$ and bifidobacteria are also indirectly involved in the production of equol, facilitating the formation of precursor metabolites or favoring the presence of equol-producing bacteria. The administration of Lactobacillus gasseri influences the effect of isoflavonoids on the host, probably through changes in the gastrointestinal environment [114].

2.2.2. Lignans, Aging, and Probiotic Bacteria. Lignans, which are the major phytoestrogens occurring in Western diets, have relevant health properties [115]. However, plant lignans are not usually absorbed and must be metabolized to enterodiol and enterolactone prior to absorption $[67,116]$. These compounds are the main responsible agents for the beneficial effects of lignans [117]. The transformation of plant lignans by intestinal microbiota is essential for the manifestation of these functions [118]. Enterolignans could be used in ameliorating some menopausal symptoms, protecting against atherosclerotic plaque deposition and due to their hepatoprotective effects [119-122].

Deglycosylation of the secoisolariciresinol diglucoside (SDG) present in the lignan extracts into secoisolariciresinol (SECO) is the first step towards the formation of enterolignans. The production of SECO from lignan extracts and SDG is widespread within LAB and bifidobacteria isolates $[78,123]$. SDG hydrolysis is an important feature in probiotic bacteria to enhance the release of SECO, improving its bioavailability for absorption by colonic mucosa and/or the biotransformation to enterodiol and enterolactone by intestinal microorganisms $[118,124]$.

Nowadays, different bacteria such as Butyribacterium methylotrophicum, Eubacterium callanderi, and Peptostreptococcus productus and the strains Eubacterium limosum, Ruminococcus productus, Clostridium scindens, Peptostreptococcus productus SECO-Mt75m3, and Eggerthella lenta SECO-Mt75m 2 have been involved in the production of enterolignans $[65,118]$. Recently, we have described the first probiotic bacterium (B. adolescentis INIA P784) capable of metabolizing lignan extracts to produce enterodiol, being the first time that the production of enterolignans by a unique bacterium strain is registered [78].

2.2.3. Ellagitannins, Aging, and Probiotic Bacteria. Ellagitannins are complex derivatives of ellagic acid, which are largely metabolized by the colon microbiota of different mammals $[125,126]$ and humans prior to absorption $[127,128]$. The microbially mediated origin of urolithin has been demonstrated [129, 130]. Ellagitannins, ellagic acid, and urolithins exhibit anticancer properties in vitro and in vivo $[69,131]$. Pomegranate extracts inhibit the growth of lung, prostate, colon, and breast cancer cells in vitro [132-135]. Urolithins inhibit mitogen-activated protein kinase signalling [136], which could curtail the risk of development of colon cancer by inhibiting cell proliferation and inducing apoptosis [90].

To date, only two urolithin-producing strains, Gordonibacter urolithinfaciens CEBAS 1/15P and Gordonibacter pamelaeae DSM 19378, have been identified [137, 138]. However, these strains cannot produce the downstream products urolithin A and urolithin B. Unraveling the bacterial phyla or group of bacteria responsible for production of these compounds is of great interest since they can be potentially used as probiotics [139]. Consumption of foods containing ellagic acid is also associated with health beneficial effects, and they could be mediated by the presence of urolithin-producing microorganisms [77].

Probiotics able to produce or to increase species related to the production of urolithins or other phytoestrogens such as equol and enterolignans can mean a step forward in the probiotic interventions, increasing the bioavailability of these compounds, and subsequently their therapeutic applications.

\section{Conclusion}

Age-related changes in nutritional behaviour and microbial diversity during aging result in a higher susceptibility to infections and diseases. Likewise, the presence of some beneficial microorganisms in the gut could help to prevent or delay some age-associated diseases by improving the immune response, or by the production of bioactive metabolites as equol, enterolignans, and urolithins. The evidence for intake of probiotics along with age specifically oriented diet to improve the health during aging is promising. However, further studies for a rational manipulation of the gut microbiota are needed to better define the role of probiotics and to assess the real potential of these interventions.

\section{Conflicts of Interest}

The authors declare that there are no conflicts of interest regarding the publication of this paper.

\section{Acknowledgments}

Financial support from the Spanish Ministry of Economy and Competitiveness (MINECO), Projects RM12-00004-0000 and RTA2013-00029-00-00, is acknowledged.

\section{References}

[1] D. Gems and L. Partridge, "Genetics of longevity in model organisms: Debates and paradigm shifts," Annual Review of Physiology, vol. 75, pp. 621-644, 2013.

[2] C. López-Otín, M. A. Blasco, L. Partridge, M. Serrano, and G. Kroemer, “The hallmarks of aging," Cell, vol. 153, no. 6, pp. 11941217, 2013. 
[3] T. Von Zglinicki, "Replicative senescence and the art of counting," Experimental Gerontology, vol. 38, no. 11-12, pp. 1259-1264, 2003.

[4] R. Ostan, L. Bucci, M. Capri et al., "Immunosenescence and immunogenetics of human longevity," NeuroImmunoModulation, vol. 15, no. 4-6, pp. 224-240, 2008.

[5] C. Franceschi, "Inflammaging as a major characteristic of old people: can it be prevented or cured?" Nutrition Reviews, vol. 65, no. 3, pp. S173-S176, 2007.

[6] E. Cevenini, D. Monti, and C. Franceschi, "Inflamm-ageing," Current opinion in clinical nutrition and metabolic care, vol. 16, pp. 14-20, 2013.

[7] C. Lee and V. Longo, "Dietary restriction with and without caloric restriction for healthy aging," F1000Research, vol. 5, 2016.

[8] M. J. Claesson, S. Cusack, O. O'Sullivan et al., "Composition, variability, and temporal stability of the intestinal microbiota of the elderly," Proceedings of the National Academy of Sciences of the United States of America, vol. 108, pp. 4586-4591, 2011.

[9] L. A. David, C. F. Maurice, R. N. Carmody et al., "Diet rapidly and reproducibly alters the human gut microbiome," Nature, vol. 505, pp. 559-563, 2014.

[10] M. Kumar, P. Babaei, B. Ji, and J. Nielsen, "Human gut microbiota and healthy aging: Recent developments and future prospective," Nutrition and Healthy Aging, vol. 4, no. 1, pp. 3-16, 2016.

[11] S. Carding, K. Verbeke, D. T. Vipond, B. M. Corfe, and L. J. Owen, "Dysbiosis of the gut microbiota in disease," Microbial Ecology in Health \& Disease, vol. 26, p. 26191, 2015.

[12] M. J. Saffrey, "Aging of the mammalian gastrointestinal tract: A complex organ system," Age, vol. 36, pp. 1019-1032, 2014.

[13] A. L. Man, E. Bertelli, S. Rentini et al., "Age-associated modifications of intestinal permeability and innate immunity in human small intestine," Clinical Science, vol. 129, no. 7, pp. 515-527, 2015.

[14] F. Rodier, J. Coppe, C. K. Patil et al., "Persistent DNA damage signalling triggers senescence-associated inflammatory cytokine secretion," Nature Cell Biology, vol. 11, pp. 973-979, 2009.

[15] T. Clairembault, L. Leclair-Visonneau, E. Coron et al., "Structural alterations of the intestinal epithelial barrier in Parkinson's disease," Acta neuropathologica communications, vol. 3, pp. 12$18,2015$.

[16] B. Yacyshyn, J. Meddings, D. Sadowski, and M. B. BowenYacyshyn, "Multiple sclerosis patients have peripheral blood CD45RO+ B cells and increased intestinal permeability," Digestive Diseases and Sciences, vol. 41, no. 12, pp. 2493-2498, 1996.

[17] C. Nicoletti, "Age-associated changes of the intestinal epithelial barrier: Local and systemic implications," Expert Review of Gastroenterology and Hepatology, vol. 9, no. 12, pp. 1467-1469, 2015.

[18] M. Rera, R. I. Clark, and D. W. Walker, "Intestinal barrier dysfunction links metabolic and inflammatory markers of aging to death in Drosophila," Proceedings of the National Academy of Sciences of the United States of America, vol. 109, no. 52, pp. 21528-21533, 2012.

[19] M. Rera, M. J. Azizi, and D. W. Walker, "Organ-specific mediation of lifespan extension: More than a gut feeling?" Ageing Research Reviews, vol. 12, no. 1, pp. 436-444, 2013.

[20] B. Lakshminarayanan, C. Stanton, P. W. O’Toole, and R. P. Ross, "Compositional dynamics of the human intestinal microbiota with aging: implications for health," The journal of nutrition, health \& aging, vol. 18, no. 9, pp. 773-786, 2014.
[21] J. Pragnesh, M. D. Patel, K. Shailesh, M. D. Singh, M. D. Siddak Panaich, and L. Cardozo, "The aging gut and the role of prebiotics, probiotics, and synbiotics: A review," Journal of Clinical Gerontology and Geriatrics, vol. 5, pp. 3-6, 2014.

[22] E. Biagi, L. Nylund, M. Candela et al., "Through ageing, and beyond: Gut microbiota and inflammatory status in seniors and centenarians," PLoS ONE, vol. 5, Articale ID e10667, 2010.

[23] M. J. Claesson, I. B. Jeffery, S. Conde et al., "Gut microbiota composition correlates with diet and health in the elderly," Nature, vol. 488, pp. 178-184, 2012.

[24] E. Cevenini, C. Caruso, G. Candore et al., "Age-related inflammation: the contribution of different organs, tissues and systems. How to face it for therapeutic approaches," Current Pharmaceutical Design, vol. 16, no. 6, pp. 609-618, 2010.

[25] E. Biagi, M. Candela, S. Fairweather-Tait, C. Franceschi, and P. Brigidi, "Ageing of the human metaorganism: The microbial counterpart," Age, vol. 34, no. 1, pp. 247-267, 2012.

[26] N. Kamada, G. Y. Chen, N. Inohara, and G. Núñez, "Control of pathogens and pathobionts by the gut microbiota," Nature Immunology, vol. 14, no. 7, pp. 685-690, 2013.

[27] M. Hornef, "Pathogens, commensal symbionts, and pathobionts: Discovery and functional effects on the host," ILAR Journal, vol. 56, no. 2, pp. 159-162, 2015.

[28] S. P. Claus and J. R. Swann, "Nutrimetabonomics: Applications for nutritional sciences, with specific reference to gut microbial interactions," Annual Review of Food Science and Technology, vol. 4, no. 1, pp. 381-399, 2013.

[29] T. Rescigno, L. Micolucci, M. F. Tecce, and A. Capasso, "Bioactive nutrients and nutrigenomics in age-related diseases," Molecules, vol. 22, no. 1, p. 105, 2017.

[30] S. Collino, I. Montoliu, F.-P. J. Martin et al., "Metabolic Signatures of Extreme Longevity in Northern Italian Centenarians Reveal a Complex Remodeling of Lipids, Amino Acids, and Gut Microbiota Metabolism," PLoS ONE, vol. 8, Article ID e56564, 2013.

[31] N. G. Rossen, J. K. MacDonald, E. M. de Vries et al., "Fecal microbiota transplantation as novel therapy in gastroenterology: a systematic review," World Journal of Gastroenterology, vol. 21, no. 17, pp. 5359-5371, 2015.

[32] E. O. Petrof, G. B. Gloor, S. J. Vanner et al., "Stool substitute transplant therapy for the eradication of Clostridium difficile infection: "RePOOPulating" the gut," Microbiome, vol. 1, pp. 38, 2013.

[33] J. M. T. Hamilton-Miller, "Probiotics and prebiotics in the elderly," Postgraduate Medical Journal, vol. 80, no. 946, pp. 447451, 2004.

[34] G. Malaguarnera, F. Leggio, M. Vacante et al., "Probiotics in the gastrointestinal diseases of the elderly," Journal of Nutrition, Health and Aging, vol. 16, no. 4, pp. 402-410, 2012.

[35] W. B. Ershler and E. T. Keller, "Age-associated increased interleukin-6 gene expression, late-life diseases, and frailty," Annual Review of Medicine, vol. 51, pp. 245-270, 2000.

[36] S. C. Bischoff, G. Barbara, W. Buurman et al., "Intestinal permeability - a new target for disease prevention and therapy," BMC Gastroenterology, vol. 14, p. 189, 2014.

[37] G. Reid, J. Jass, M. T. Sebulsky, and J. K. McCormick, "Potential uses of probiotics in clinical practice," Clinical Microbiology Reviews, vol. 16, no. 4, pp. 658-672, 2003.

[38] H. Fang, T. Elina, A. Heikki, and S. Seppo, "Modulation of humoral immune response through probiotic intake," FEMS Immunology \& Medical Microbiology, vol. 29, no. 1, pp. 47-52, 2006. 
[39] A. T. Borchers, C. Selmi, F. J. Meyers, C. L. Keen, and M. E. Gershwin, "Probiotics and immunity," Journal of Gastroenterology, vol. 44, no. 1, pp. 26-46, 2009.

[40] J. M. Otte and D. K. Podolsky, "Functional modulation of enterocytes by gram-positive and gram-negative microorganisms," The American Journal of Physiology-Gastrointestinal and Liver Physiology, vol. 286, no. 4, p. G613, 2004.

[41] M. D. Luyer, W. A. Buurman, M. Hadfoune et al., "Strainspecific effects of probiotics on gut barrier integrity following hemorrhagic shock," Infection and Immunity, vol. 73, no. 6, pp. 3686-3692, 2005.

[42] S. C. Ng, A. L. Hart, M. A. Kamm, A. J. Stagg, and S. C. Knight, "Mechanisms of action of probiotics: recent advances," Inflammatory Bowel Diseases, vol. 15, no. 2, pp. 300-310, 2009.

[43] R. C. Anderson, A. L. Cookson, W. C. McNabb et al., "Lactobacillus plantarum MB452 enhances the function of the intestinal barrier by increasing the expression levels of genes involved in tight junction formation," BMC Microbiology, vol. 10, pp. 316-327, 2010.

[44] J. Karczewski, F. J. Troost, I. Konings et al., "Regulation of human epithelial tight junction proteins by Lactobacillus plantarum in vivo and protective effects on the epithelial barrier," American Journal of Physiology - Gastrointestinal and Liver Physiology, vol. 298, no. 6, pp. G851-G859, 2010.

[45] M. Matsumoto, S. Kurihara, R. Kibe, H. Ashida, and Y. Benno, "Longevity in mice is promoted by probiotic-induced suppression of colonic senescence dependent on upregulation of gut bacterial polyamine production," PLoS ONE, vol. 6, Article ID e23652, 2011.

[46] R. Sultana, A. J. McBain, and C. A. O’Neill, “Strain-dependent augmentation of tight-junction barrier function in human primary epidermal keratinocytes by lactobacillus and bifidobacterium lysates," Applied and Environmental Microbiology, vol. 79, no. 16, pp. 4887-4894, 2013.

[47] L. Stewart, B. Crumley, and K. Walton, "Effects of probiotic VSL\#3 on cytokine and tight junction protein expression in intestinal epithelial cells," FASEB-The Federation of American Societies for Experimental Biology, vol. 29, 1010.4, 2015.

[48] L. Malaguarnera, E. Cristaldi, and M. Malaguarnera, "The role of immunity in elderly cancer," Critical Reviews in Oncology/Hematology, vol. 74, no. 1, pp. 40-60, 2010.

[49] H. S. Gill, M. L. Cross, K. J. Rutherfurd, and et al, "Dietary probiotic supplementation to enhance cellular immunity in the elderly," Br J BiomedSci-British Journal of Biomedical Science, vol. 58, pp. 94-96, 2001.

[50] H. S. Gill, K. J. Rutherfurd, and M. L. Cross, "Dietary probiotic supplementation enhances natural killer cell activity in the elderly: An investigation of age-related immunological changes," Journal of Clinical Immunology, vol. 21, no. 4, pp. 264$271,2001$.

[51] H. S. Gill, K. J. Rutherfurd, M. L. Cross, and P. K. Gopal, "Enhancement of immunity in the elderly by dietary supplementation with the probiotic Bifidobactedum lactis HN019," Am J Clin Nutr-American Journal of Clinical Nutrition, vol. 74, no. 6, pp. 833-839, 2001.

[52] H. Kimoto-Nira, C. Suzuki, M. Kobayashi, K. Sasaki, J. Kurisaki, and K. Mizumachi, "Anti-ageing effect of a lactococcal strain: Analysis using senescence-accelerated mice," Br J Nutr-British Journal of Nutrition, vol. 98, no. 6, pp. 1178-1186, 2007.

[53] R. Sharma, R. Kapila, G. Dass, and S. Kapila, "Improvement in Th1/Th2 immune homeostasis, antioxidative status and resistance to pathogenic E. coli on consumption of probiotic Lactobacillus rhamnosus fermented milk in aging mice," Age, vol. 36, pp. 9686-9703, 2014.

[54] H.-Y. Yang, S.-L. Liu, S. A. Ibrahim et al., "Oral administration of live Bifidobacterium substrains isolated from healthy centenarians enhanced immune function in BALB/c mice," Nutrition Research, vol. 29, no. 4, pp. 281-289, 2009.

[55] K. Vidal, J. Benyacoub, M. Moser et al., "Effect of Lactobacillus paracasei NCC2461 on antigen-specific T-cell mediated immune responses in aged mice," Rejuvenation Research, vol. 11, no. 5, pp. 957-964, 2008.

[56] V. Molina, M. Médici, J. Villena, G. Font, and M. P. Taranto, "Dietary Supplementation with Probiotic Strain Improves Immune-Health in Aged Mice," Open J Immunol-Open Journal of Immunology, vol. 6, no. 3, pp. 73-78, 2016.

[57] A. A. van Beek, B. Sovran, F. Hugenholtz et al., "Supplementation with lactobacillus plantarum wcfs1 prevents decline of mucus barrier in colon of accelerated aging Erccl-/ $\Delta 7$ mice," Frontiers in Immunology, vol. 7, p. 408, 2016.

[58] K. Arunachalam, H. S. Gill, and R. K. Chandra, "Enhancement of natural immune function by dietary consumption of Bifidobacterium lactis (HN019)," European Journal of Clinical Nutrition, vol. 54, no. 3, pp. 263-267, 2000.

[59] B. L. Chiang, Y. H. Sheih, L. H. Wang, C. K. Liao, and H. S. Gill, "Enhancing immunity by dietary consumption of a probiotic lactic acid bacterium (Bifidobacterium lactis HN019): Optimization and definition of cellular immune responses," Eur J Clin Nutr-European Journal of Clinical Nutrition, vol. 54, no. 11, pp. 849-855, 2000.

[60] F. Ibrahim, S. Ruvio, L. Granlund, S. Salminen, M. Viitanen, and A. C. Ouwehand, "Probiotics and immunosenescence: Cheese as a carrier," FEMS Immunology and Medical Microbiology, vol. 59, no. 1, pp. 53-59, 2010.

[61] P. Turchet, M. Laurenzano, S. Auboiron, and J. M. Antoine, "Effect of fermented milk containing the probiotic Lactobacillus casei DN-114001 on winter infections in free-living elderly subjects: A randomised, controlled pilot study," J Nutr Health Aging-Journal of Nutrition, Health and Aging, vol. 7, pp. 75-77, 2003.

[62] Y. Fukushima, S. Miyaguchi, T. Yamano et al., "Improvement of nutritional status and incidence of infection in hospitalised, enterally fed elderly by feeding of fermented milk containing probiotic Lactobacillus johnsonii Lal (NCC533)," Br J Nutr-British Journal of Nutrition, vol. 98, no. 5, pp. 969-977, 2007.

[63] H.-J. Wang and P. A. Murphy, "Isoflavone content in commercial soybean foods," J Agric Food Chem-Journal of Agricultural and Food Chemistry, vol. 42, no. 8, pp. 1666-1673, 1994.

[64] J. M. Landete, "Ellagitannins, ellagic acid and their derived metabolites: a review about source, metabolism, functions and health," Food Research International, vol. 44, no. 5, pp. 1150-1160, 2011.

[65] J. M. Landete, "Plant and mammalian lignans: a review of source, intake, metabolism, intestinal bacteria and health," Food Research International, vol. 46, no. 1, pp. 410-424, 2012.

[66] D. M. Harris, E. Besselink, S. M. Henning, V. L. W. Go, and D. Heber, "Phytoestrogens induce differential estrogen receptor alpha- or beta-mediated responses in transfected breast cancer cells," Exp Biol Med_Experimental Biology and Medicine, vol. 230, no. 8, pp. 558-568, 2005. 
[67] J. M. Landete, J. L. Arqués, M. Medina, P. Gaya, B. D. de Las Rivas, and R. Muñoz, "Bioactivation of Phytoestrogens: Intestinal Bacteria and Health," Crit Rev Food Sci Nutr-Critical Reviews in Food Science and Nutrition, vol. 56, no. 11, pp. 18261843, 2016.

[68] L.-J. W. Lu, J. A. Tice, and F. L. Bellino, "Phytoestrogens and healthy aging: Gaps in knowledge. A workshop report," Menopause, vol. 8, no. 3, pp. 157-170, 2001.

[69] N. P. Seeram, W. J. Aronson, Y. Zhang et al., "Pomegranate ellagitannin-derived metabolites inhibit prostate cancer growth and localize to the mouse prostate gland," J Agric Food Chem-Journal of Agricultural and Food Chemistry, vol. 55, no. 19, pp. 7732-7737, 2007.

[70] Y. H. Chan, T. H. Larn, K. K. Lau et al., "Dietary intake of phytoestrogen is associated with increased circulating endothelial progenitor cells in patients with cardiovascular disease," Eur J Prevent Cardiol-European Journal of Cardiovascular Prevention and Rehabilitation, vol. 18, no. 3, pp. 360-368, 2011.

[71] Y. Yeo, K. P. Ko, S. H. Ma et al., "Abstract 4823: Isoflavones from phytoestrogens and colorectal cancer risk: A nested casecontrol study within the Korean Multicenter Cancer Cohort," Cancer Research, vol. 73, no. 8, p. 4823, 2014.

[72] E. Poluzzi, C. Piccinni, E. Raschi, A. Rampa, M. Recanatini, and F. de Ponti, "Phytoestrogens in postmenopause: the state of the art from a chemical, pharmacological and regulatory perspective," Current Medicinal Chemistry, vol. 21, pp. 417-436, 2014.

[73] M. N. Chen, C. C. Lin, and C. F. Liu, "Efficacy of phytoestrogens for menopausal symptoms: a meta-analysis and systematic review," Climacteric, vol. 18, pp. 260-269, 2015.

[74] S. Bolca, T. van de Wiele, and S. Possemiers, "Gut metabotypes govern health effects of dietary polyphenols," Current Opinion in Biotechnology, vol. 24, no. 2, pp. 1-6, 2012.

[75] D. Bialonska, P. Ramnani, S. G. Kasimsetty, K. R. Muntha, G. R. Gibson, and D. Ferreira, "The influence of pomegranate byproduct and punicalagins on selected groups of human intestinal microbiota," International Journal of Food Microbiology, vol. 140, no. 2-3, pp. 175-182, 2010.

[76] Y. Shimada, S. Yasuda, M. Takahashi et al., "Cloning and expression of a novel NADP(H)-dependent daidzein reductase, an enzyme involved in the metabolism of daidzein, from equolproducing Lactococcus strain 20-92," Applied and Environmental Microbiology, vol. 76, no. 17, pp. 5892-5901, 2010.

[77] M. Romo-Vaquero, R. García-Villalba, A. González-Sarrías et al., "Interindividual variability in the human metabolism of ellagic acid: Contribution of Gordonibacter to urolithin production," Journal of Functional Foods, vol. 17, pp. 785-791, 2015.

[78] P. Gaya, A. Peirotén, M. Medina, and J. M. Landete, "Bifidobacterium adolescentis INIA P784: The first probiotic bacterium capable of producing enterodiol from lignan extracts," Journal of Functional Foods, vol. 29, pp. 269-274, 2017.

[79] A. Scalbert and G. Williamson, "Dietary intake and bioavailability of polyphenols," J Nutr-Journal of Nutrition, vol. 130, pp. 2073-2085, 2000.

[80] J. M. Landete, J. A. Curiel, H. Rodríguez, B. de las Rivas, and R. Muñoz, "Aryl glycosidases from Lactobacillus plantarum increase antioxidant activity of phenolic compounds," Journal of Functional Foods, vol. 7, no. 1, pp. 322-329, 2014.

[81] K. Morito, T. Hirose, J. Kinjo et al., "Interaction of phytoestrogens with estrogen receptors $\alpha$ and $\beta$," Biological and Pharmaceutical Bulletin, vol. 24, no. 4, pp. 351-356, 2001.
[82] Y.-L. Low, J. I. Taylor, P. B. Grace et al., “Phytoestrogen exposure correlation with plasma estradiol in postmenopausal women in European Prospective Investigation of Cancer and Nutrition-Norfolk may involve diet-gene interactions," Cancer Epidemiology Biomarkers and Prevention, vol. 14, pp. 213-220, 2005.

[83] M. S. Touillaud, P. C. Pillow, J. Jakovljevic et al., "Effect of dietary intake of phytoestrogens on estrogen receptor status in premenopausal women with breast cancer," Nutrition and Cancer, vol. 51, no. 2, pp. 162-169, 2005.

[84] A. M. Hutchins, M. C. Martini, B. A. Olson, W. Thomas, and J. L. Slavin, "Flaxseed consumption influences endogenous hormone concentrations in postmenopausal women," Nutrition and Cancer, vol. 39, no. 1, pp. 58-65, 2001.

[85] C. S. Hwang, H. S. Kwaka, H. J. Lim et al., "Isoflavone metabolites and their in vitro dual functions: They can act as an estrogenic agonist or antagonist depending on the estrogen concentration," Journal of Steroid Biochemistry and Molecular Biology, vol. 101, no. 4-5, pp. 246-253, 2006.

[86] J. D. Brooks, W. E. Ward, J. E. Lewis et al., "Supplementation with flaxseed alters estrogen metabolism in postmenopausal women to a greater extent than does supplementation with an equal amount of soy," Am J Clin Nutr-American Journal of Clinical Nutrition, vol. 79, pp. 318-325, 2004.

[87] D. D. Kitts, Y. V. Yuan, A. N. Wijewickreme, and L. U. Thompson, "Antioxidant activity of the flaxseed lignan secoisolariciresinol diglycoside and its mammalian lignan metabolites enterodiol and enterolactone," Molecular \& Cellular Biochemistry, vol. 202, no. 1-2, pp. 91-100, 1999.

[88] K. Prasad, "Antioxidant activity of secoisolariciresinol diglucoside-derived metabolites, secoisolariciresinol, enterodiol, and enterolactone," International Journal of Angiology, vol. 9, no. 4, pp. 220-225, 2000.

[89] Z. Juranic, Z. Zizak, S. Tasic et al., "Antiproliferative action of water extracts of seeds or pulp of five different raspberry cultivars," Food Chemistry, vol. 93, no. 1, pp. 39-45, 2005.

[90] S. G. Kasimsetty, D. Bialonska, M. K. Reddy, G. Ma, S. I. Khan, and D. Ferreira, "Colon cancer chemopreventive activities of pomegranate ellagitannins and urolithins," Journal of Agricultural and Food Chemistry, vol. 58, no. 4, pp. 2180-2187, 2010.

[91] P. Gaya, A. Peiroten, M. Medina, and J. M. Landete, "Isoflavone metabolism by a collection of lactic acid bacteria and bifidobacteria with biotechnological interest," Int J Food Sci Technol-International Journal of Food Sciences and Nutrition, vol. 67, no. 2, pp. 117-124, 2016.

[92] H.-G. Hur, J. O. Lay Jr., R. D. Beger, J. P. Freeman, and F. Rafii, "Isolation of human intestinal bacteria metabolizing the natural isoflavone glycosides daidzin and genistin," Archives of Microbiology, vol. 174, no. 6, pp. 422-428, 2000.

[93] D. B. Fournier, J. W. Erdman Jr., and G. B. Gordon, "Soy, its components, and cancer prevention: a review of the in vitro, animal, and human data," Cancer Epidem Biomar, vol. 7, pp. 1055-1065, 1998.

[94] M. Messina, O. Kucuk, and J. W. Lampe, "An Overview of the health effects of isoflavones and emphasis on prostate cancer risk and prostate-specific antigen levels," J AOAC Int-Journal of AOAC International, vol. 89, pp. 1121-1134, 2006.

[95] K. K. Han, J. M. Soares, M. A. Haidar, G. R. de Lima, and E. Baracat, "Benefits of soy isoflavone therapeutic regimen on menopausal symptoms," Obstetrics \& Gynecology, vol. 99, pp. 389-394, 2002.

[96] J. W. Erdman Jr., "Soy protein and cardiovascular disease: A statement for healthcare professionals from the nutrition 
committee of the AHA," Circulation, vol. 102, no. 20, pp. 25552559, 2000.

[97] M. Messina, E. T. Gugger, and Alekel D. L., "Soy protein, soybean isoflavones, and bone health," in Handbook of Nutraceuticals and Functional Foods (Wildman, R., ed.), pp. 77-98, CRC Press, Boca Raton, FL, 2001.

[98] D. Kritz-Silverstein, D. Von Mühlen, E. Barrett-Connor, and M. Bressel, "Isoflavones and cognitive function in older women: The soy and postmenopausal health in aging (SOPHIA) study," Menopause, vol. 10, no. 3, pp. 196-202, 2003.

[99] J. Chun, G. M. Kim, K. W. Lee et al., "Conversion of isoflavone glucosides to aglycones in soymilk by fermentation with lactic acid bacteria," Journal of Food Science, vol. 72, no. 2, pp. 39-44, 2007.

[100] J. A. Marazza, M. S. Garro, and de Giori G. S., "Aglycone production by Lactobacillus rhamnosus CRL981 during soymilk fermentation," Food Microbiology, vol. 26, no. 3, pp. 333-339, 2009.

[101] X.-L. Wang, H.-G. Hur, J. H. Lee, K. T. Kim, and S.-I. Kim, "Enantioselective synthesis of S-equol from dihydrodaidzein by a newly isolated anaerobic human intestinal bacterium," Applied and Environmental Microbiology, vol. 71, no. 1, pp. 214-219, 2005.

[102] K. D. R. Setchell, "Equol - Origins, actions, and clinical relevance of this specific soy isoflavone metabolite," J Nutr-Journal of Nutrition, vol. 134, pp. 1235S-1236S, 2004.

[103] E. D. Lephart, "Skin aging and oxidative stress: Equol's antiaging effects via biochemical and molecular mechanisms," Ageing Research Reviews, vol. 31, pp. 36-54, 2016.

[104] L. Subedi, E. Ji, D. Shin, J. Jin, J. H. Yeo, and S. Y. Kim, "Equol, a dietary daidzein gut metabolite attenuates microglial activation and potentiates neuroprotection in vitro," Nutrients, vol. 9, no. 3, p. 207, 2017.

[105] P. J. Magee, M. Raschke, C. Steiner et al., "Equol: A comparison of the effects of the racemic compound with that of the purified S-enantiomer on the growth, invasion, and DNA integrity of breast and prostate cells in vitro," Nutrition and Cancer, vol. 54, no. 2, pp. 232-242, 2006.

[106] T. Aso, "Equol improves menopausal symptoms in Japanese women," Journal of Nutrition, vol. 140, no. 7, pp. 1386S-1389S, 2010.

[107] Z.-M. Liu, S. C. Ho, Y.-M. Chen et al., "Whole soy, but not purified daidzein, had a favorable effect on improvement of cardiovascular risks: A 6-month randomized, double-blind, and placebo-controlled trial in equol-producing postmenopausal women," Molecular Nutrition and Food Research, vol. 58, no. 4, pp. 709-717, 2013.

[108] D. Ingram, K. Sanders, M. Kolybaba, and D. Lopez, "Casecontrol study of phyto-oestrogens and breast cancer," The Lancet, vol. 350, no. 9083, pp. 990-994, 1997.

[109] C. L. Frankenfeld, "O-Desmethylangolensin: The importance of equol's lesser known cousin to human health," Advances in Nutrition, vol. 2, no. 4, pp. 317-324, 2011.

[110] K. Decroos, S. Vanhemmens, S. Cattoir, N. Boon, and W. Verstraete, "Isolation and characterisation of an equol-producing mixed microbial culture from a human faecal sample and its activity under gastrointestinal conditions," Archives of Microbiology, vol. 183, no. 1, pp. 45-55, 2005.

[111] X.-L. Wang, H.-J. Kim, S.-I. Kang, S.-I. Kim, and H.-G. Hur, "Production of phytoestrogen S-equol from daidzein in mixed culture of two anaerobic bacteria," Archives of Microbiology, vol. 187, no. 2, pp. 155-160, 2007.
[112] S. Uchiyama, T. Ueno, and T. Suzuki, "Identification of a newly isolated equol-producing lactic acid bacterium from the human feces," J Intest Microbiol-Journal of Intestinal Microbiology, vol. 21, pp. 217-220, 2007.

[113] S. Elghali, S. Mustafa, M. Amid, M. Y. A. Manap, A. Ismail, and F. Abas, "Bioconversion of daidzein to equol by Bifidobacterium breve 15700 and Bifidobacterium longum BB536," Journal of Functional Foods, vol. 4, no. 4, pp. 736-745, 2012.

[114] M. Tamura, M. Ohnishi-Kameyama, and K. Shinohara, "Lactobacillus gasseri: Effects on mouse intestinal flora enzyme activity and isoflavonoids in the caecum and plasma," British Journal of Nutrition, vol. 92, no. 5, pp. 771-776, 2004.

[115] M. J. J. de Klejin, Y. T. der Schouw van, P. W. F. Wilson et al., "Intake of dietary phytoestrogens is low in postmenopausal women in the United States: The Framingham Study," J Nutr-Journal of Nutrition, vol. 131, pp. 1826-1832, 2001.

[116] A. Quartieri, R. García-Villalba, A. Amaretti et al., "Detection of novel metabolites of flaxseed lignans in vitro and in vivo," Molecular Nutrition and Food Research, vol. 60, no. 7, pp. 15901601, 2016.

[117] A. L. Edel, G. N. Pierce, and M. Aliani, "Age-dependency in the metabolism of flaxseed lignans by healthy adults," Journal of Functional Foods, vol. 17, pp. 948-957, 2015.

[118] T. Clavel, G. Henderson, C.-A. Alpert et al., "Intestinal bacterial communities that produce active estrogen-like compounds enterodiol and enterolactone in humans," Applied and Environmental Microbiology, vol. 71, no. 10, pp. 6077-6085, 2005.

[119] D. Del Rio, A. Rodriguez-Mateos, J. P. E. Spencer, M. Tognolini, G. Borges, and A. Crozier, "Dietary (poly)phenolics in human health: structures, bioavailability, and evidence of protective effects against chronic diseases," Antioxidants \& Redox Signaling, vol. 18, no. 14, pp. 1818-1892, 2013.

[120] K. B. Gutte, A. K. Sahoo, and R. C. Ranveer, "Bioactive components of flaxseed and its health benefits," Int J PharmSci Rev Res-International Journal of Pharmaceutical Sciences Review and Research, vol. 31, pp. 42-51, 2015.

[121] S. C. Yoder, S. M. Lancaster, M. A. J. Hullar, and J. W. Lampe, "Gut Microbial Metabolism of Plant Lignans: Influence on Human Health," Diet-Microbe Interactions in the Gut: Effects on Human Health and Disease, pp. 103-117, 2015.

[122] C. M. C. Dupasquier, A.-M. Weber, B. P. Ander et al., "Effects of dietary flaxseed on vascular contractile function and atherosclerosis during prolonged hypercholesterolemia in rabbits," American Journal of Physiology - Heart and Circulatory Physiology, vol. 291, no. 6, pp. H2987-H2996, 2006.

[123] L. Roncaglia, A. Amaretti, S. Raimondi, A. Leonardi, and M. Rossi, "Role of bifidobacteria in the activation of the lignan secoisolariciresinol diglucoside," Applied Microbiology and Biotechnology, vol. 92, no. 1, pp. 159-168, 2011.

[124] T. Clavel, R. Lippman, F. Gavini, J. Doré, and M. Blaut, “Clostridium saccharogumia sp. nov. and Lactonifactor longoviformis gen. nov., sp. nov., two novel human faecal bacteria involved in the conversion of the dietary phytoestrogen secoisolariciresinol diglucoside," Systematic and Applied Microbiology, vol. 30, no. 1, pp. 16-26, 2007.

[125] B. Cerdá, R. Llorach, J. J. Cerón, J. C. Espín, and F. A. TomásBarberán, "Evaluation of the bioavailability and metabolism in the rat of punicalagin, an antioxidant polyphenol from pomegranate juice," European Journal of Nutrition, vol. 42, no. 1, pp. 18-28, 2003.

[126] J. C. Espín, R. González-Barrio, B. Cerdá, C. López-Bote, A. I. Rey, and F. A. Tomás-Barberán, "Iberian pig as a model to 
clarify obscure points in the bioavailability and metabolism of ellagitannins in humans," Journal of Agricultural and Food Chemistry, vol. 55, no. 25, pp. 10476-10485, 2007.

[127] B. Cerdá, J. C. Espín, S. Parra, P. Martínez, and F. A. TomásBarberán, "The potent in vitro antioxidant ellagitannins from pomegranate juice are metabolised into bioavailable but poor antioxidant hydroxy-6H-dibenzopyran-6-one derivatives by the colonic microflora of healthy humans," European Journal of Nutrition, vol. 43, no. 4, pp. 205-220, 2004.

[128] M. Larrosa, A. González-Sarrías, M. T. García-Conesa, F. A. Tomás-Barberán, and J. C. Espín, "Urolithins, ellagic acidderived metabolites produced by human colonic microflora, exhibit estrogenic and antiestrogenic activities," Journal of Agricultural and Food Chemistry, vol. 54, no. 5, pp. 1611-1620, 2006.

[129] B. Cerdá, P. Periago, J. C. Espín, and F. A. Tomás-Barberán, "Identification of urolithin A as a metabolite produced by human colon microflora from ellagic acid and related compounds," Journal of Agricultural and Food Chemistry, vol. 53, no. 14, pp. 5571-5576, 2005.

[130] H. Ito, A. Iguchi, and T. Hatano, "Identification of urinary and intestinal bacterial metabolites of ellagitannin geraniin in rats," Journal of Agricultural and Food Chemistry, vol. 56, no. 2, pp. 393-400, 2008.

[131] D. Heber, "Multitargeted therapy of cancer by ellagitannins," Cancer Letters, vol. 269, no. 2, pp. 262-268, 2008.

[132] N. D. Kim, R. Mehta, W. Yu et al., "Chemopreventive and adjuvant therapeutic potential of pomegranate (Punica granatum) for human breast cancer," Breast Cancer Research and Treatment, vol. 71, no. 3, pp. 203-217, 2002.

[133] A. Malik, F. Afaq, S. Sarfaraz, V. M. Adhami, D. N. Syed, and H. Mukhtar, "Pomegranate fruit juice for chemoprevention and chemotherapy of prostate cancer," Proceedings of the National Academy of Sciences of the United States of America, vol. 102, no. 41, pp. 14813-14818, 2005.

[134] N. P. Seeram, L. S. Adams, S. M. Henning et al., "In vitro antiproliferative, apoptotic and antioxidant activities of punicalagin, ellagic acid and a total pomegranate tannin extract are enhanced in combination with other polyphenols as found in pomegranate juice," Journal of Nutritional Biochemistry, vol. 16, no. 6, pp. 360-367, 2005.

[135] M. Larrosa, F. A. Tomás-Barberán, and J. C. Espín, “The dietary hydrolysable tannin punicalagin releases ellagic acid that induces apoptosis in human colon adenocarcinoma Caco-2 cells by using the mitochondrial pathway," Journal of Nutritional Biochemistry, vol. 17, no. 9, pp. 611-625, 2006.

[136] A. González-Sarrías, M. Azorín-Ortuño, M.-J. Yáñez-Gascón, F. A. Tomás-Barberán, M.-T. García-Conesa, and J.-C. Espín, "Dissimilar in vitro and in vivo effects of ellagic acid and its microbiota-derived metabolites, urolithins, on the cytochrome P450 1A1," Journal of Agricultural and Food Chemistry, vol. 57, no. 12, pp. 5623-5632, 2009.

[137] M. V. Selma, F. A. Tomás-Barberán, D. Beltrán, R. GarcíaVillalba, and J. C. Espín, "Gordonibacter urolithinfaciens sp. nov., a urolithin-producing bacterium isolated from the human gut," International Journal of Systematic and Evolutionary Microbiology, vol. 64, no. 7, pp. 2346-2352, 2014.

[138] M. V. Selma, D. Beltran, R. Garcia-Villalba, J. C. Espin, and F. A. Tomas-Barberan, "Description of urolithin production capacity from ellagic acid of two human intestinal Gordonibacter species," Food Funct, vol. 5, pp. 1779-1784, 2014.
[139] P. Saha, B. S. Yeoh, R. Singh et al., "Gut Microbiota conversion of dietary ellagic acid into bioactive phytoceutical urolithin a inhibits heme peroxidases," PLoS ONE, vol. 11, Article ID e0156811, no. 6, 2016. 

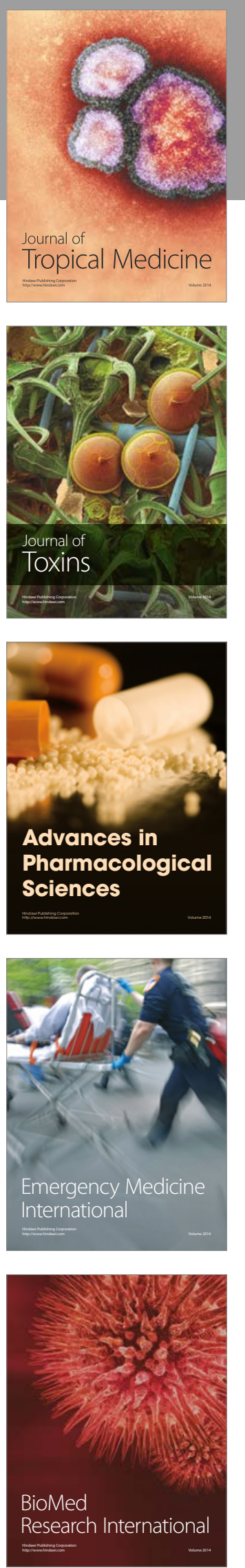
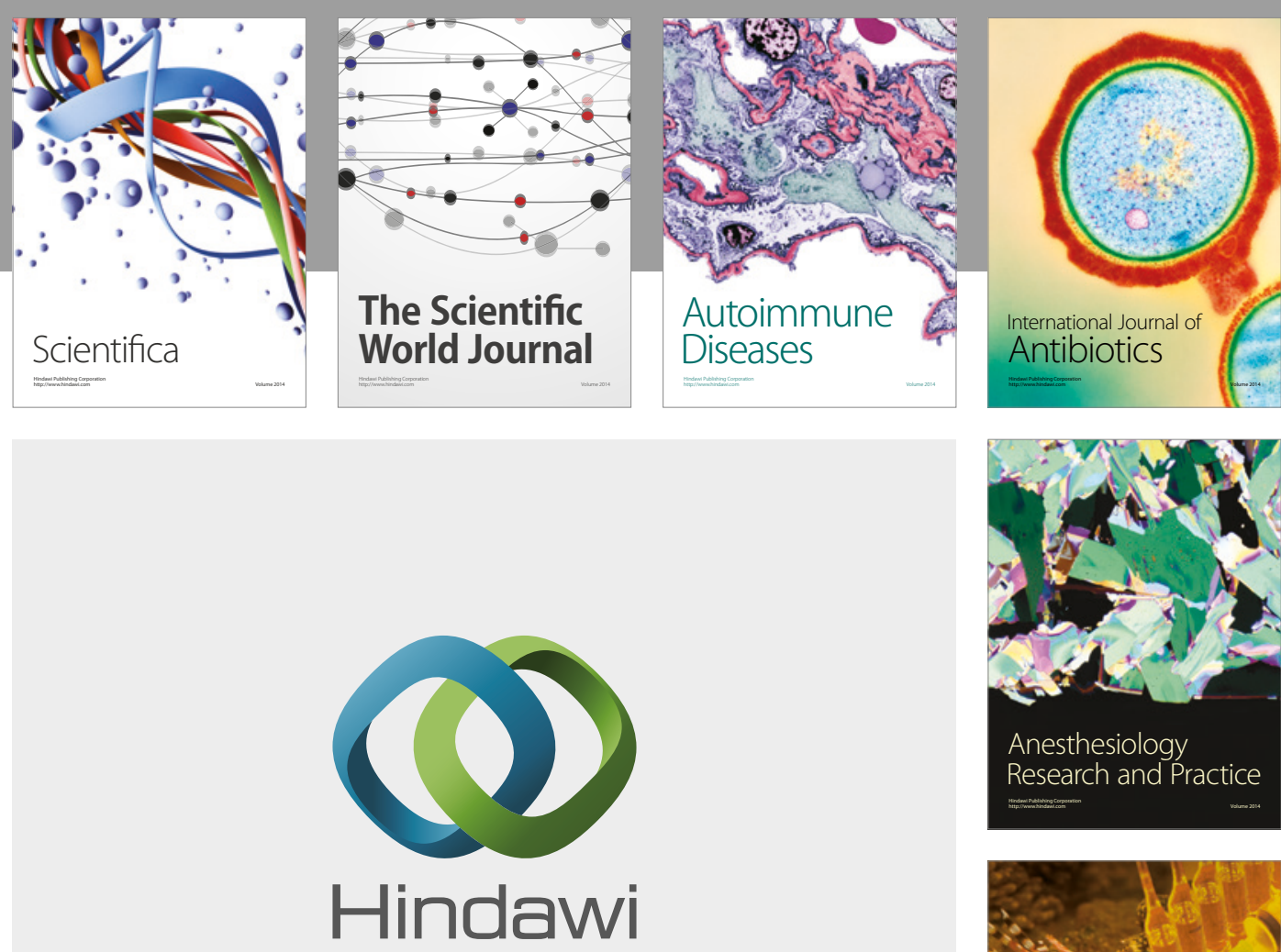

Submit your manuscripts at

https://www.hindawi.com
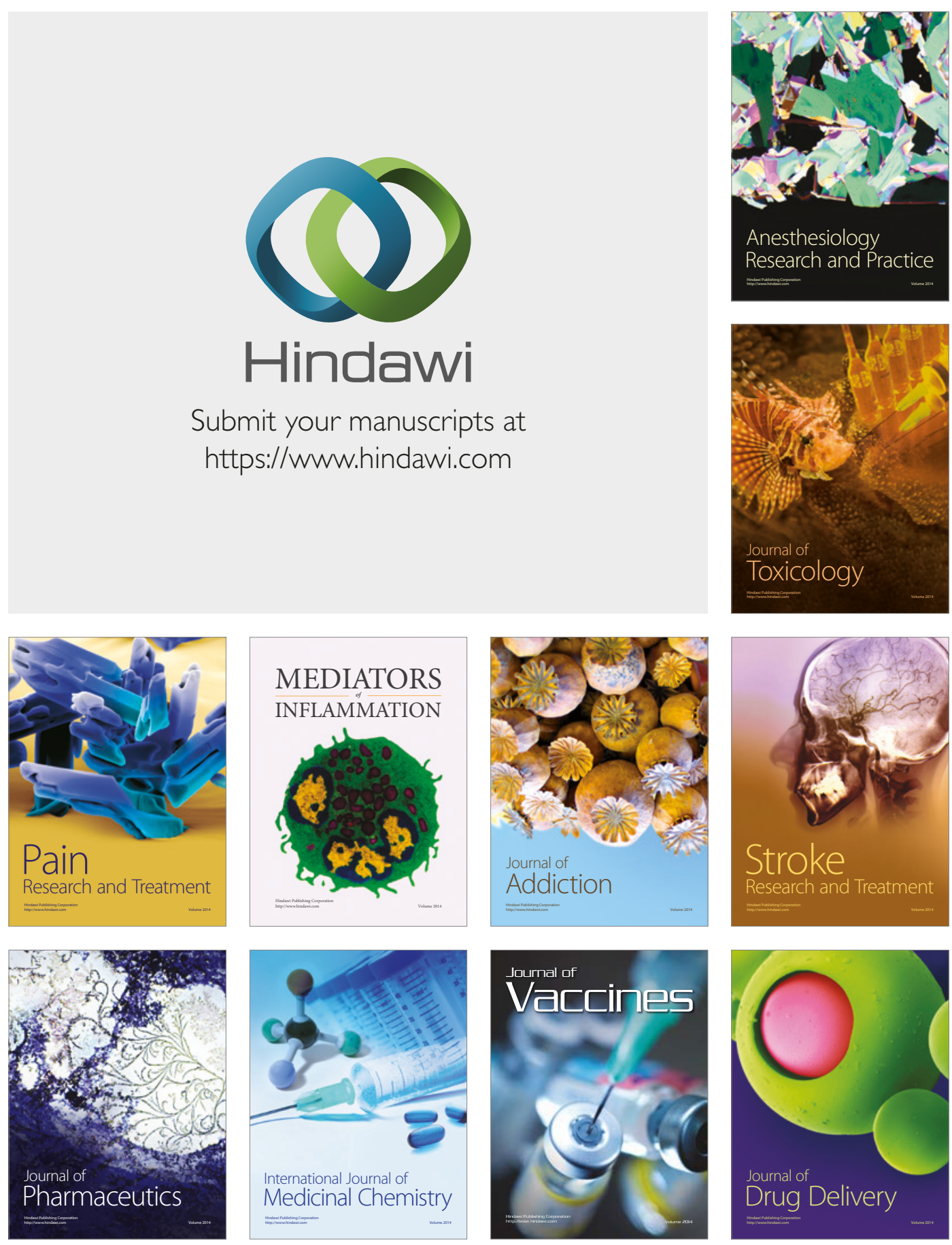